\title{
Tratamento do lagoftalmo paralítico com a utilização do implante de peso de ouro recoberto pela aponeurose do músculo levantador palpebral
}

\section{Paralytic lagophthalmos treatment with gold weight implants covered by levator palpebrae muscle aponeurosis}

Sergio Lessa', Marcelo Nanci', Eduardo Flores ${ }^{3}$, Roberto Sebastiáa

\section{ResUMO}

Objetivo: Demonstrar a redução das complicações e melhora do resultado estético da correção do lagoftalmo paralítico com utilização de implantes de peso ouro através do recobrimento pela aponeurose do músculo levantador palpebral. Métodos: Foram estudados vinte e nove pacientes portadores de paralisia facial idiopática com evolução clínica superior a 4 anos submetidos à correção cirúrgica do lagoftalmo paralítico com a utilização de uma modificação da técnica do implante de peso de ouro recoberto pela aponeurose do músculo levantador palpebral (Gladstone,1996) entre junho de 1997 e dezembro de 2006. Nenhum deles havia se submetido previamente a tratamentos cirúrgicos. Resultados: Todos os pacientes apresentaram adaptação favorável dos implantes. Não houve casos de extrusão, deslocamento ou infecção, com melhora significativa da sintomatologia clínica e redução do lagoftalmo paralítico no período de acompanhamento pós-operatório que variou de 8 meses a 4 anos. Conclusão: Através da ampla dissecção da aponeurose do músculo levantador palpebral para recobrimento completo do implante do peso de ouro nós obtivemos excelentes resultados. O contorno da superfície cutânea pré-tarsal apresentou-se homogêneo, evitou-se a extrusão do implante e, comparativamente aos resultados apresentados pela técnica inicialmente descrita por Gladstone na qual realizava-se apenas a plicatura da aponeurose do músculo levantador palpebral sobre o implante de ouro, obtivemos um melhor controle do posicionamento final da pálpebra superior após o recobrimento do implante.

Descritores: Paralisia facial/complicações; Doenças palpebrais/etiologia; Doenças palpebrais/ cirurgia; Ouro; Procedimentos cirúrgicos oftalmológicos/métodos

\footnotetext{
'Professor Assistente do Curso de Pós-graduação Médica da Pontifícia Universidade Católica do Rio de Janeiro - PUCRJ - Rio de Janeiro (RJ); do Instituto de Pós-graduação Médica Carlos Chagas, $38^{a}$ Enfermaria da Santa Casa da Misericórdia do Rio de Janeiro - SCMRJ Rio de Janeiro (RJ) e Chefe do Departamento de Cirurgia Plástica Ocular da $1^{\text {a }}$ e $38^{\mathrm{a}}$. Enfermaria da SCMRJ - Rio de Janeiro (RJ), Brasil; ${ }^{2}$ Cirurgião Membro do Departamento de Plástica Ocular da $1^{\text {a }}$ Enfermaria da Santa Casa da Misericórdia do Rio de Janeiro - SCMRJ Rio de Janeiro (RJ), Brasil;

Professor Assistente do Curso de Pós-graduação Médica da Pontifícia Universidade Católica do Rio de Janeiro - PUCRJ - Rio de Janeiro (RJ); do Instituto de Pós-graduação Médica Carlos Chagas, $38^{a}$ Enfermaria da Santa Casa da Misericórdia do Rio de Janeiro - SCMRJ Rio de Janeiro (RJ), Brasil;

${ }^{4}$ Professor Assistente do Curso de Pós-graduação Médica da Pontifícia Universidade Católica do Rio de Janeiro - PUCRJ - Rio de Janeiro (RJ); do Instituto de Pós-Graduação Médica Carlos Chagas, $38^{a}$ Enfermaria da Santa Casa da Misericórdia do Rio de Janeiro - SCMRJ; Professor Adjunto do Departamento de Plástica Ocular do Serviço de Oftalmologia do Hospital Universitário Antônio Pedro da Universidade Federal Fluminense - UFF - Rio de Janeiro (RJ), Brasil.
} 


\section{INTRODUÇÃO}

$\mathbf{L}$ agoftalmo e ceratite por exposição constituem sequelas comuns da paralisia facial ${ }^{(1,2)}$ (Figura1). A inadequada proteção ocular conduz a ulcerações na córnea e muitas vezes à perfuração ocu$\operatorname{lar}^{(1,2)}$. O tratamento inicial inclui lubrificação ocular, oclusão da fenda palpebral, uso de câmara úmida e medidas para aumentar o grau de umidade ambiental.

As medidas cirúrgicas tradicionalmente envolvem tarsorrafias mediais e laterais ${ }^{(1.2)}$. Infelizmente, muitas dessas técnicas não produzem os resultados desejados, obstruindo a visão periférica, não promovendo proteção corneana adequada e gerando grandes distorções da anatomia da fenda palpebral.

Retalhos dos músculos temporal e masseter foram largamente empregados ${ }^{(3-6)}$. Estas técnicas possuem a desvantagem de alterar a anatomia da fenda palpebral, além de não permitirem um piscamento espontâneo, o que diminui radicalmente a distribuição do filme lacrimal e a lubrificação ocular. São técnicas complexas e que muitas vezes necessitam de reoperações.

Uma segunda categoria de técnicas cirúrgicas inclui a reanimação palpebral com utilização de implantes. Arion, em $1972^{(1)}$, e posteriormente Lessa e Carreirão, em $1978{ }^{(8)}$, utilizaram a cerclagem palpebral com fios de silicone para facilitar a oclusão palpebral. Esta técnica entrou em desuso devido às dificuldades de equilíbrio entre a tensão aliada à elasticidade do fio e a força de elevação do músculo levantador da pálpebra superior. Em alguns casos, ocorria alteração da forma da fenda palpebral, principalmente com distorções do canto medial ${ }^{(8)}$.

Morel-Fatio e Lalardrie ${ }^{(9)}$ descreveram o uso do implante da mola metálica para fechamento da fenda palpebral. Esta técnica foi abandonada, pois eram necessárias frequentes reoperações para corrigir o deslocamento da mola ${ }^{(8)}$. Modificações nesta técnica foram introduzidas por Levine ${ }^{(1)}$ e May ${ }^{(1)}$, com melhora dos resultados cosméticos e funcionais, porém foram descritos inúmeros casos de extrusão e dificuldade de ajuste técnico da mola, limitando sua utilização ${ }^{(12)}$.

A técnica de fechamento dinâmico da fenda palpebral utilizando implantes metálicos foi descrita na literatura norte-americana, em 1950, por Sheehan ${ }^{(13)}$. A inclusão do peso de ouro nas pálpebras paralisadas foi inicialmente descrita por Illig, em $1958^{(14)}$, e, recentemente, amplamente divulgada por Smellie ${ }^{(15)}$, Jobe ${ }^{(16)} \mathrm{e}$ May ${ }^{(17)} \mathrm{e}$ assim o recobrimento dos implantes passou a ser utilizado com objetivo de reduzir as complicações

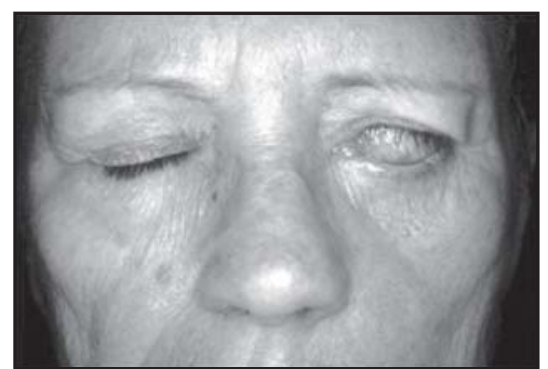

Figura 1: Paciente com 65 anos e paralisia facial à esquerda apresentando lagoftalmo paralítico

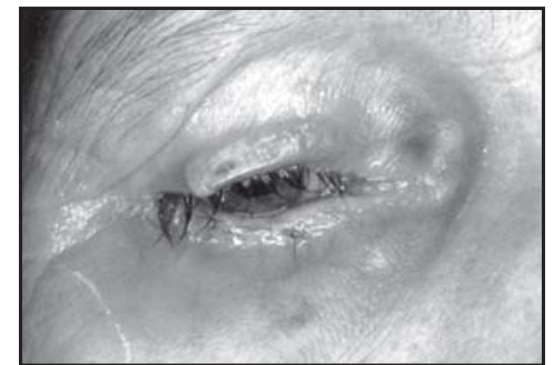

Figura 2: Extrusão parcial do peso de ouro em pálpebra superior direita

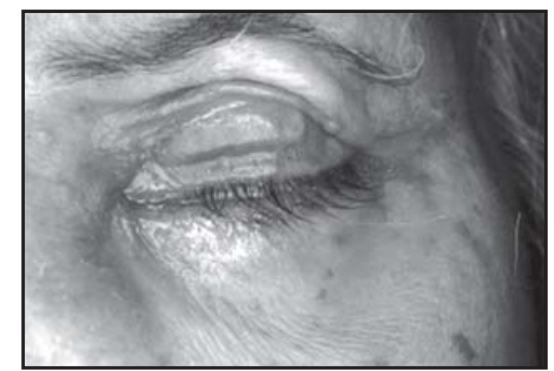

Figura 3: Visibilidade do peso de ouro através da pele palpebral

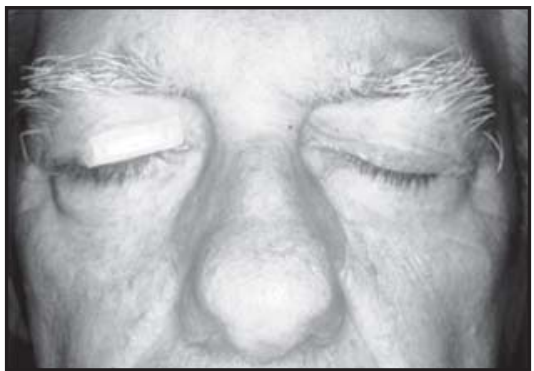

Figura 4: Fixação dos pesos para teste às pálpebras superiores com tiras de Micropore ${ }^{\circledR}$ em posição logo acima dos cílios na junção do terço medial com o terço central da pálpebra superior

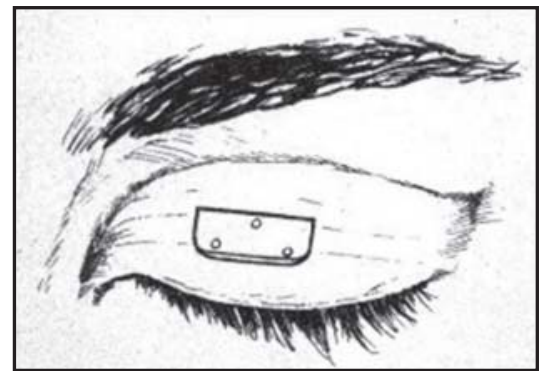

Figura 5: Esquema demonstrando o local ideal para posicionamento do peso de ouro 


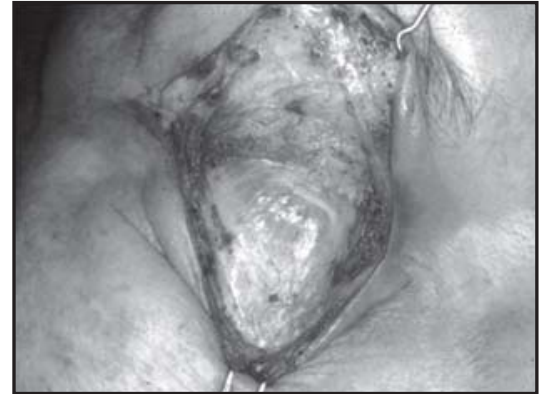

Figura 6A: Incisão cutânea palpebral superior ao nível do sulco palpebral e dissecção através da lamela anterior até a placa tarsal

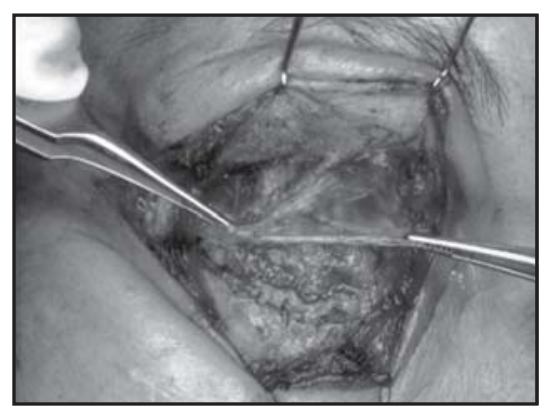

Figura 6D: Desinserção e liberação da aponeurose do músculo levantador palpebral superior da placa tarsal

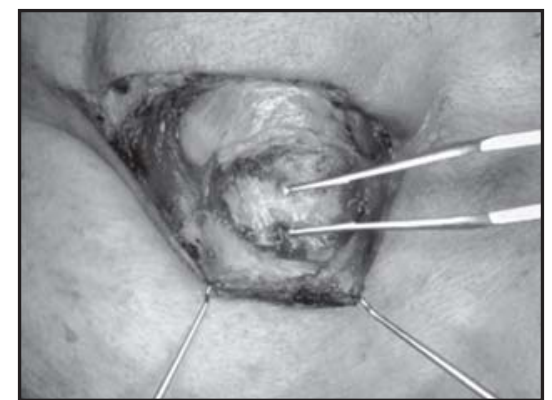

Figura 6B: Dissecção do tarso com liberação das adesões ao músculo orbicular pré-tarsal até atingir a área próxima à raiz dos cílios

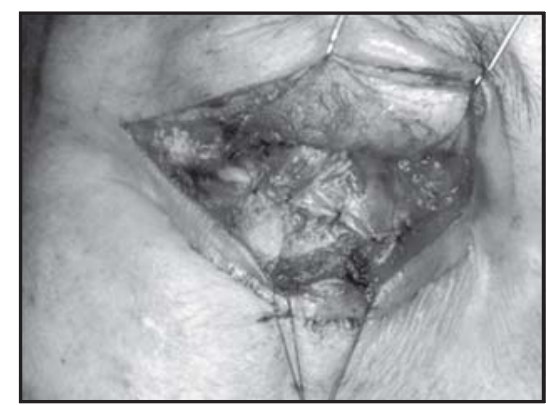

Figura 6E: Aponeurose do músculo levantador palpebral suturada ao tarso inferiormente, sobre o peso de ouro

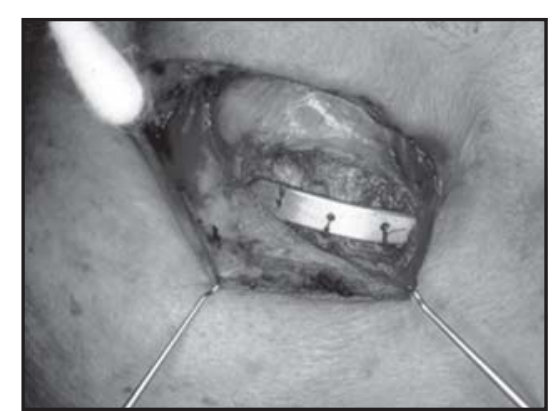

Figura 6C: Posicionamento supratarsal do peso de ouro. Suturas absorvíveis de poliglactina 6-0 são posicionadas através dos orifícios de fixação localizados no implante fixando-o ao nível da junção do terço medial com o terço central da distância horizontal da pálpebra inferior

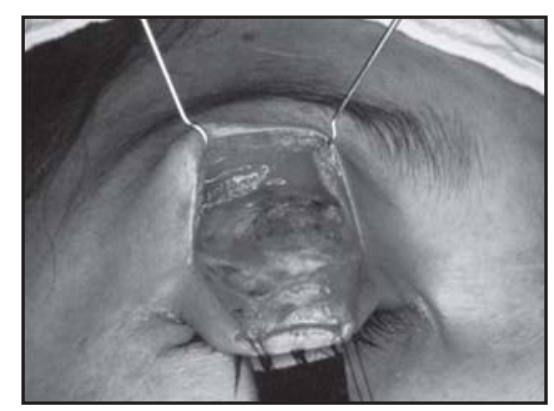

Figura 6F: Alongamento da aponeurose do músculo levantador palpebral após realização das miotomias marginais das técnicas tradicionais onde o implante era recoberto apenas pelo músculo orbicular. Uma grande variedade de materiais autólogos e aloplásticos passaram a ser utilizados, tais como telas absorvíveis e não-absorvíveis, fáscia temporal, fáscia lata e pericárdio humano processado laboratorialmente ${ }^{(18-21)}$.

O implante do peso de ouro utilizado no tratamento do lagoftalmo paralítico é $99.95 \%$ puro e apresenta peso adequado para promover a oclusão da fenda palpebral. A função normal do músculo levantador palpebral é responsável pela abertura da pálpebra superior. Este procedimento é relativamente simples mas apresenta um grande número de complicações associadas, tais como extrusão do implante (Figura 2), visibilidade do implante através da pele fina palpebral (Figura 3) e ptose palpebral ${ }^{(1,2)}$.

O presente trabalho objetiva demonstrar que através da utilização de uma modificação da técnica de Gladstone ${ }^{(12)}$ de recobrimento do implante com a aponeurose do músculo levantador palpebral, conseguimos reduzir essas complicações, obtendo fechamento dinâmico da fenda palpebral com concomitante resultado estético satisfatório em casos de paralisia facial.

\section{Métodos}

Vinte e nove pacientes foram submetidos a tratamento cirúrgico para reanimação das pálpebras paralisadas entre junho de 1997 e dezembro de 2006, utilizando a técnica do implante de peso de ouro recoberto pela aponeurose do músculo levantador palpebral.

Os pacientes apresentavam paralisia facial idiopática com evolução superior a 4 anos. A idade dos pacientes variou de 37 a 75 anos, sendo 10 do sexo masculino e 19 do sexo feminino. Doze pacientes apresentavam paralisia à esquerda e dezessete pacientes à direita. Todos os pacientes apresentavam lagoftalmo com ceratopatia e irritação ocular não adequadamente controlados pela adoção de medidas conservadoras. Nenhum dos pacientes havia se submetido a cirurgias prévias.

Os implantes de ouro selecionados apresentavam 99.95\% de grau de pureza, minimizando os riscos de 


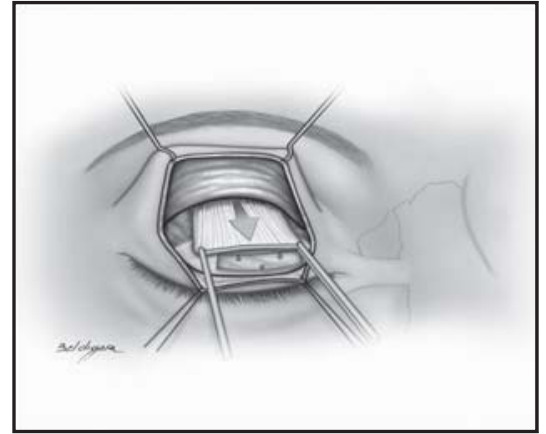

Figura 7A: Esquema demonstrando o avançamento da aponeurose do levantador palpebral sobre o peso de ouro

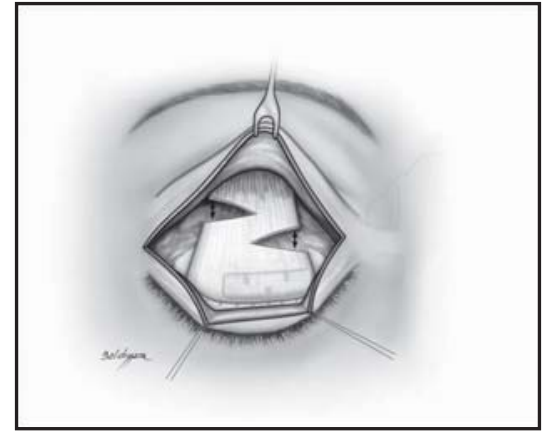

Figura 7B: Apresentação das miotomias lateral e marginal sobre a aponeurose do levantador palpebral

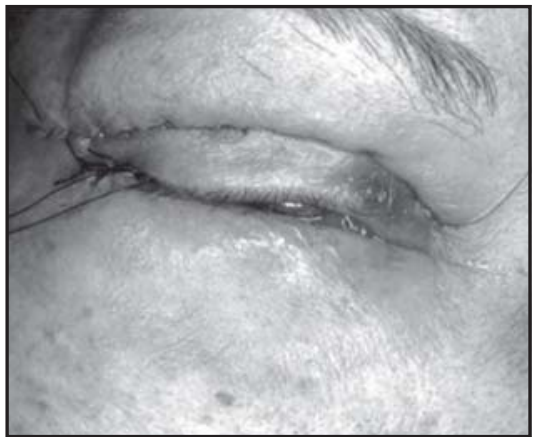

Figura 8: Aspecto final - Implante de peso de ouro em pálpebra superior e retalho tarsal em pálpebra inferior reações alérgicas aos implantes. As peças de ouro apresentavam $1 \mathrm{~mm}$ de espessura e 4,5 mm de altura. Apresentavam dois ou mais orifícios para permitir a perfeita fixação com suturas e suas bordas eram abauladas, evitando as arestas. $\mathrm{O}$ comprimento variava com o peso dos implantes, cujos valores foram de 0,8 a 1,6 gramas.

\section{Técnica cirúrgica}

O peso dos implantes é determinado previamente à operação, testando-se diferentes peças sobre a pálpebra superior. Com o paciente sentado o peso é fixado com uma fina tira de Micropore ${ }^{\circledR}$ ou usando cola especial (Figura 4), logo acima dos cílios, na junção do terço medial com o terço central da pálpebra, no ponto onde a função do músculo levantador palpebral é maior ${ }^{(16)}(\mathrm{Fi}-$ gura 5).

O peso apropriado deve permitir a completa oclusão da fenda palpebral sem ocasionar ptose palpebral superior maior que $2 \mathrm{~mm}$ quando os olhos estiverem abertos. As pálpebras inferiores são examinadas e caso apresentem alterações de posição ou flacidez excessiva, também realiza-se a abordagem cirúrgica desses segmentos.

A cirurgia é realizada sob anestesia local utilizando lidocaína $2 \%$ associada à epinefrina com concentração de 1:200.000 UI. A incisão cutânea é posicionada ao longo do sulco palpebral superior (Figura 6A) e a dissecção prossegue através das camadas da lamela anterior até atingir o tarso, que é então liberado do músculo orbicular pré-tarsal, atingindo a área próxima da raiz dos cílios (Figura 6B). O implante é colocado sobre região anterior do tarso previamente exposto na junção entre os terços medial e central da pálpebra superior, e aí fortemente fixado através de pontos passados pelos orifícios estrategicamente posicionados na peça de ouro (Figura 6C). Nesta fase o peso é avaliado em relação à posição e a seu perfeito contorno. Um discreto grau de ptose pode estar presente, condição que espontaneamente é resolvida com o retorno da função do músculo levantador palpebral.

A dissecção avança proximalmente ao nível do septo orbital, que é incisado, seguindo cranialmente para exposição da aponeurose do músculo levantador palpebral. A aponeurose é desinserida do tarso e dissecada pelo plano profundo por aproximadamente 1 $\mathrm{cm}$. Incisões medial e lateral são realizadas na aponeurose para permitir um perfeito avançamento sobre o peso de ouro (Figuras 6D e 7A). Nesta fase, a extremidade liberada da aponeurose é suturada sobre o tarso inferiormente, recobrindo integralmente o peso de ouro (Figura 6E).

O paciente é então avaliado, acordado e em posição sentada, pois o avançamento da aponeurose provoca uma retração palpebral superior. Deve-se então proceder a uma liberação ampla das adesões ao septo orbital para se evitar a ocorrência de lagoftalmo restritivo futuro. A retração da pálpebra superior é solucionada com miotomias realizadas na aponeurose do músculo levantador, semelhantes às utilizadas para tratamento das retrações palpebrais superiores na Oftalmopatia de Graves ${ }^{(22)}$ : uma incisão aponeurótica medial é cuidadosamente feita a $5 \mathrm{~mm}$ acima do rebordo tarsal superior, e outra, em posição paralela, a $5 \mathrm{~mm}$ de distância da anterior, lateralmente (Figuras 6F e 7B). A extensão das incisões aponeuróticas vai variar com a resolução da retração palpebral, devendo a pálpebra superior permanecer numa posição "não-ptótica".

A pele é finalmente fechada com sutura contínua. Não empregamos curativo oclusivo e utilizamos compressas frias nas primeiras 48 horas.

O reparo do ectrópio paralítico da pálpebra inferior é realizado no mesmo tempo cirúrgico com uma 


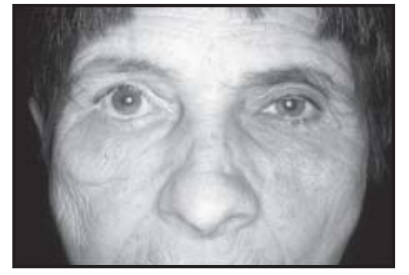

Figura 9A: Mulher de 64 anos de idade com lagoftalmo paralítico e ectrópio palpebral inferior à direita

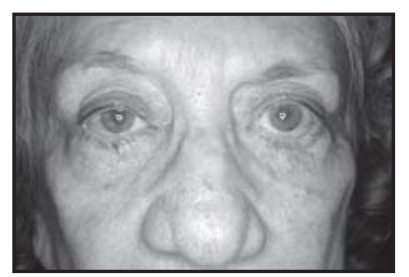

Figura 10A: Mulher de 72 anos de idade com lagoftalmo paralítico e ectrópio palpebral inferior à esquerda

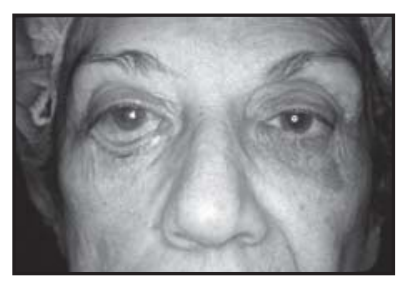

Figura 11A: Mulher de 75 anos de idade com lagoftalmo paralítico e ectrópio palpebral inferior à direita

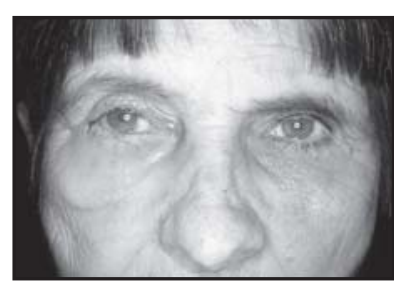

Figura 9B: Reanimação palpebral dinâmica com implante de peso de ouro e retalho tarsal inferior

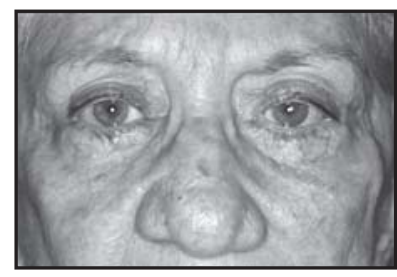

Figura 10B: Reanimação palpebral dinâmica com implante de peso de ouro e cantoplastia de suspensão tarsal inferior

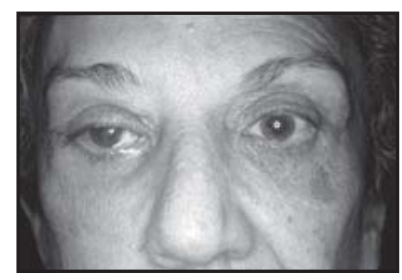

Figura 11B: Reanimação palpebral dinâmica com implante de peso de ouro e retalho tarsal inferior

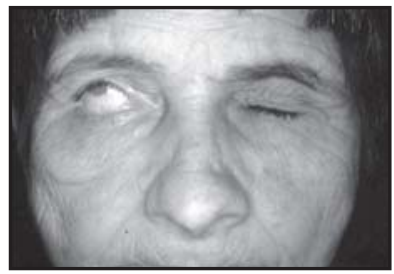

Figura 9C: Mulher de 64 anos de idade com lagoftalmo paralítico e ectrópio palpebral inferior à direita

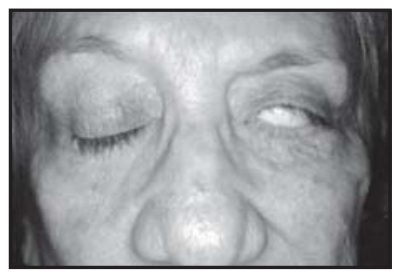

Figura 10C: Mulher de 72 anos de idade com lagoftalmo paralítico e ectrópio palpebral inferior à esquerda

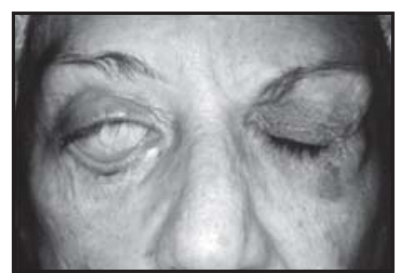

Figura 11C: Mulher de 75 anos de idade com lagoftalmo paralítico e ectrópio palpebral inferior à direita

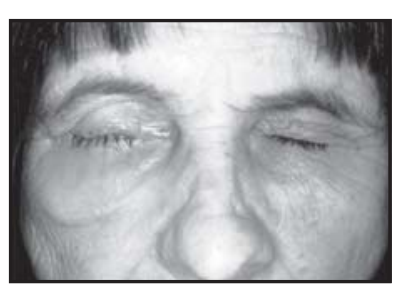

Figura 9D: Reanimação palpebral dinâmica com implante de peso de ouro e retalho tarsal inferior. Oclusão completa da fissura palpebral

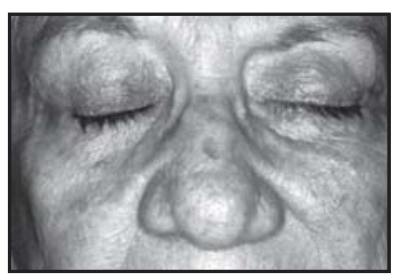

Figura 10D: Reanimação palpebral dinâmica com implante de peso de ouro e cantoplastia de suspensão tarsal inferior. Oclusão completa da fissura palpebral

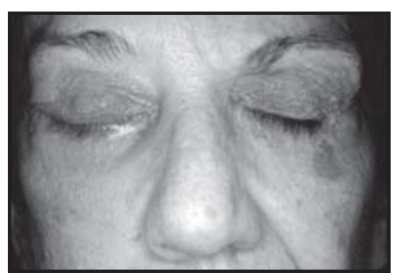

Figura 11D: Reanimação palpebral dinâmica com implante de peso de ouro e retalho tarsal inferior. Oclusão completa da fissura palpebral

cantoplastia lateral com retalho tarsal e secção dos retratores inferiores (Figura 8) ou apenas com uma cantoplastia por suspensão tarsal. Eventualmente, podemos associar autoenxertia de cartilagem conchal auricular nos casos de graves alterações de posição das pálpebras inferiores.

\section{Resultados}

Nenhuma complicação grave foi observada. Todos os pacientes mantiveram os implantes, isto é, não foi observado nenhum caso de extrusão, deslocamento ou infecção (Figuras 9, 10 e 11).

O período de acompanhamento pós-operatório variou de 8 meses a 4 anos. $O$ peso dos implantes utilizados variou de 0,8 a 1,4 g, média de 1,2 g. Os pacientes

apresentaram significativa melhora da ceratopatia por exposição, com redução do lagoftalmo.

Cinco pacientes $(17,2 \%)$ apresentaram edema persistente pós-operatório, que se resolveu espontaneamente após 3 meses da cirurgia. Um paciente $(3,4 \%)$ apresentou edema persistente que involuiu completamente após 2 anos. Discreta retração palpebral superior foi observada em dois pacientes $(6,8 \%)$ e os mesmos necessitaram de nova miotomia do levantador palpebral no sexto mês pós-operatório. Um paciente $(3,4 \%)$ apresentou ptose palpebral de $2 \mathrm{~mm}$. Por se tratar de uma paciente idosa (75 anos) que não apresentava sintomatologia que a incomodasse ou queixas estéticas, optou-se por descartar a reoperação. Um paciente $(3,4 \%)$ apresentou visibilidade do implante através da pele após o primeiro ano da cirurgia. 


\section{Discussão}

As técnicas que utilizam implantes do peso de ouro para tratamento do lagoftalmo paralítico vêm apresentando aceitação crescente entre os cirurgiões plásticos e oftalmologistas. A técnica apresenta muitas vantagens por ser de simples execução, os implantes serem bem tolerados a curto e longo prazo e serem relativamente inertes biologicamente. Entre suas eventuais desvantagens citamos a possibilidade de infecção, deslocamentos e extrusão ${ }^{(1,2)}$.

O problema da reação tecidual ao implante foi praticamente resolvido com o uso do ouro puro, entretanto Seiff et al. ${ }^{(23)}$, Bair et al. ${ }^{(24)}$, Doyle et al. ${ }^{(25)}$ e Ritz et al. ${ }^{(26)}$ relataram reação inflamatória com edema crônico em alguns casos que necessitaram de remoção ou tratamento com infiltração local de corticóides.

A alteração inaceitável do relevo do peso de ouro sendo observado por transparência através da pele palpebral é um outro problema apresentado pelas técnicas convencionais ${ }^{(27,28)}$. Ptose palpebral é um outro achado comum ${ }^{(2,28)}$ após a simples inclusão do peso de ouro e se superior a $2 \mathrm{~mm}$ o tratamento cirúrgico está indicado.

A extrusão do implante apresenta-se como a complicação mais grave dessas técnicas ${ }^{(1,2,17,23,29)}$. Anteriormente, o peso de ouro utilizado no tratamento do lagoftalmo paralítico era recoberto pelo músculo orbicular e pele palpebral. Nos pacientes mais idosos, devido à atrofia progressiva dos tecidos, os implantes tornavam-se visíveis através da pele, comprometendo o resultado do procedimento.

O uso de materiais autólogos ou aloplásticos para recobrimento do implante vem sendo utilizado por muitos autores, porém esses materiais carregam riscos potenciais tais como reações tipo corpo estranho para telas absorvíveis, inabsorvíveis e materiais aloplásticos e morbidade da área doadora no caso dos materiais autólogos tais como fáscia temporal, fáscia lata e enxertos dérmicos. Outros materiais como pericárdio humano processado laboratorialmente requerem testes sorológicos meticulosos, bem como processamento dos tecidos doadores para prevenção da transmissão de infecções ${ }^{(18-21)}$. Tais complicações podem ser minimizadas ou resolvidas com a utilização da técnica de recobrimento do implante com a aponeurose do músculo levantador palpebral ${ }^{(12)}$, conferindo bons resultados com um relevo mais homogêneo da região pré-tarsal da pálpebra superiore evitandose deslocamentos e extrusões dos implantes.

Comparativamente aos resultados apresentados pela técnica inicialmente descrita por Gladstone ${ }^{(12)}$ na qual se realizava apenas a plicatura da aponeurose do músculo levantador palpebral sobre o implante de ouro, a ampla desinserção e dissecção da aponeurose apresentou um melhor controle do posicionamento final da pálpebra superior após o recobrimento do implante.

\section{Conclusão}

Excelentes resultados foram obtidos com a dissecção da aponeurose do músculo levantador palpebral para recobrimento completo dos implantes de peso de ouro. O contorno da superfície palpebral superior tornou-se homogêneo, evitou-se a extrusão dos implantes e promoveu-se um bom posicionamento palpebral.

Não foram realizadas ressecções de pele palpebral superior uma vez que em muitos casos, realizamos uma abordagem transpalpebral para elevação do supercílio e obtenção de um maior equilíbrio da estética periorbitária.

A utilização dos implantes de peso de ouro recobertos pela aponeurose do músculo levantador palpebral demonstrou ser uma técnica simples com baixos índices de complicações. Pequenas assimetrias de posicionamento do sulco palpebral superior podem ocorrer, entretanto sem comprometimento do resultado estético e funcional do procedimento.

A reanimação palpebral superior obtida permite uma maior proteção ocular, melhora a distribuição do filme lacrimal e sustenta o equilíbrio estético local.

\section{Abstract}

Pourpose: To demonstrate an improvement in paralytic lagophthalmos surgical treatment complications and aesthetic results by covering the gold weight implants with the levator palpebrae muscle aponeurosis.Methods: We studied a case series of twenty nine patients presenting idiopatic facial palsy with more than 4 years of clinical evolution, submitted to paralytic lagophthalmos surgical treatment using a modification of the technique of gold weight implantation covered by the aponeurosis of the levator palpebrae superioris muscle (Gladstone, 1996) between June 1997 and December 2006. None had undergone previous surgical treatment. Results: All patients sustained their implants. There were no cases of extrusion, dislocation or infection, with clinical symptoms improvement and lagophthalmos reduction. Conclusion: By widely dissecting the levator palpebrae superioris muscle aponeurosis, exceeding it and recovering the implant completely, we obtained excellent results. The pretarsal upper eyelid surface became regular, extrusion 
was avoided and, comparatively to Gladstone's technique that performs an aponeurotic plication over the gold weight implant, we managed to obtain a better adjustment of the superior eyelid final positioning after implant coverage.

Keywords: Facial palsy/complications; Eyelid diseases/etiology; Eyelid diseases/surgery; Gold; Ophthalmologic surgical procedures/methods

\section{ReFERÊNCIAS}

1. Mausolf FA. Techniques for the repair of orbicularis oculi palsy. Ophthalmic Surg. 1978;9(3):67-70.

2. Lisman RD, Smith B, Baker D, Arthurs B. Efficacy of surgical treatment of paralytic ectropion. Ophthalmology. 1987;94(6):671-81.

3. Adams WM. The use of the masseter, temporalis and frontalis muscles in the correction of facial paralysis. Plast Reconstr Surg. 1946;1(2):216.

4. Johnson HA. A modification of the Gillies temporalis transfer for the surgical treatment of the lagophthalmos of leprosy. Plast Reconstr Surg. 1962;30: 378.

5. Masters FW, Robinson DW, Simons JN. Temporalis transfer for lagophthalmos due to seventh nerve palsy. Am J Surg. 1965;110(4):607-11.

6. Baker DC, Conley J. Regional muscle transposition for rehabilitation of the paralyzed face. Clin Plast Surg. 1979;6(3):317-31.

7. Arion HG. Dynamic closure of the lids in paralysis of the orbicularis muscle. Int Surg. 1972;57(1):48-50.

8. Lessa S, Carreirao S. Use of an encircling silicone rubber string for the correction of lagophthalmos. Plast Reconstr Surg. 1978;61(5): 719-23.

9. Morel-Fatio D, Lalardrie JP. Palliative surgical treatment of facial paralysis. The palpebral spring. Plast Reconstr Surg. 1964;33:446-56.

10. Levine RE, House WF, Hitselberger WE. Ocular complications of seventh nerve paralysis and management with the palpebral spring. Am J Ophthalmol. 1972;73(2): 219-28.

11. May M. Paralyzed eyelids reanimated with a closed-eyelid spring. Laryngoscope. 1988;98(4):382-5.

12. Gladstone GJ, Nesi FA. Management of paralytic lagophthalmos with a modified gold-weight implantation technique. Ophthal Plast Reconstr Surg. 1996;12(1):38-44.

13. Sheehan JE. Progress in correction of facial palsy with tantalum wire and mesh. Surgery. 1950;27(1):122-5.

14. Illig KM. [A new method of lagophthalmos surgery]. Klin Monatsblatter Augenheilkd Augenarztl Fortbild. 1958;132(3):410-1.

15. Smellie GD. Restoration of the blinking reflex in facial palsy by a simple lid- load operation. Br J Plast Surg. 1966;19(3):279-83.
16. Jobe RP. A technique for lid loading in the management of the lagophthalmos of facial palsy. Plast Reconstr Surg. 1974;53(1):29-32.

17. May M. Gold weight and wire spring implants as alternatives to tarsorrhaphy. Arch Otolaryngol Head Neck Surg. 1987;113(6):656-60.

18. Jacob JT, Pendleton K, Broussard, Crisp A, DiLoreto DA. Porous alloplastic material encasement of gold weights for the treatment of paralytic lagophthalmos. Ophthal Plast Reconstr Surg. 1999;15(6):401-6.

19. Tremolada C, Raffaini M, D’Orto O, Gianni AB, Biglioli F, Carota F. Temporal galeal fascia cover of custom-made gold lid weights for correction of paralytic lagophthalmos: longterm evaluation of an improved technique. J Craniomaxillofac Surg. 2001;29(6):355-9.

20. Foster JA, Perry JD, Cahill KV, Holck DE, Kugler L. Processed human pericardium barrier for gold weight implantation. Ophthal Plast Reconstr Surg. 2004;20(2):107-9.

21. Thomas DA, Khalifa YM. Temporalis fascia in the management of gold eyelid weight extrusion. Ophthal Plast Reconstr Surg. 2005;21(2):153-5.

22. Grove AS Jr. Upper eyelid retraction and Graves' disease. Ophthalmology. 1981;88(6):499-506.

23. Seiff SR, Sullivan JH, Freeman LN, Ahn J. Pretarsal fixation of gold weights in facial nerve palsy. Ophthal Plast Reconstr Surg. 1989;5(2):104-9.

24. Bair RL, Harris GJ, Lyon DB, Komorowski RA. Noninfectious inflammatory response to gold weight eyelid implants. Ophthal Plast Reconstr Surg. 1995;11(3):209-14.

25. Doyle E, Mavrikakis I, Lee EJ, Emerson R, Rainey AJ, Brittain GP. Type IV hypersensitivity reactions to upper lid gold weight implants - is patch testing necessary? Orbit. 2005;24(3):205-10.

26. Ritz M, Southwick GJ, Greensmith A, Gory I. Gold sensitivity after gold weight eyelid insertion for facial palsy. Aesthetic Plast Surg. 2006;30(6):733.

27. Chapman P, Lamberty BG. Results of upper lid loading in the treatment of lagophthalmos caused by facial palsy. Br J Plast Surg. 1988;41(4):369-72.

28. Kartush JM, Linstrom CJ, McCann PM, Graham MD. Early gold weight eyelid implantation for facial paralysis. Otolaryngol Head Neck Surg. 1990;103(6):1016-23.

29. Neuman AR, Weinberg A, Sela M, Peled IJ, Wexler MR. The correction of seventh nerve palsy lagophthalmos with gold lid load (16 years experience). Ann Plast Surg. 1989;22(2):142-5.

\author{
ENDEREÇo PaRa CoRrespondência: \\ Dr. Sergio Lessa \\ Avenida Ataulfo de Paiva, $\mathrm{n}^{\circ} 135$ - Cj. 1101 \\ CEP 22449-900 - Rio de Janeiro - RJ - Brasil \\ Tel: (21) 2259-1245 - Fax: (21) 2259-0099 \\ E-mail: s.lessa@alternex.com.br
}

